\title{
Handedness and the side on which pharyngeal pouches occur
}

\author{
NICHOLAS D STAFFORD, VICTORIA MOORE-GILLON, PETER McKELVIE
}

\begin{abstract}
Pharyngeal pouches present far more commonly on the left side of the neck than the right. Sixty one patients with a history of pharyngeal pouch were questioned about their handedness and about whether they had had symptoms or signs predominantly on one side of the neck before receiving treatment. There was a highly significant association between handedness and pharyngeal pouches on the opposite side of the neck. It is suggested that this is the reason for the rarity of right sided pouches.
\end{abstract}

\section{Introduction}

Patients with pharyngeal pouches present much more commonly with left sided than right sided symptoms and signs in the neck, ${ }^{1}$ but the reason for this is not known. We had gained the impression that those rare patients presenting with pouches on the right side tended to be left handed, and so we investigated the association between handedness and the side on which pouches occur.

\section{Patients, methods, and results}

We asked 108 patients in whom pharyngeal pouch had been diagnosed and who had presented at this and two other London hospitals in the years 1966-83 to fill in a questionnaire. This asked about handedness and whether symptoms (pain, "fullness," gurgling) and signs (swelling) before treatment had occurred predominantly on one side of the neck, and if so which side. Information from returned

\footnotetext{
Royal National Throat, Nose and Ear Hospital and Institute of Laryngology and Otology, London WC1 X 8EE

NICHOLAS D STAFFORD, MB, FRCS, registrar

VICTORIA MOORE-GILLON, BSC, FRCS, research fellow

PETER MCKELVIE, MD, FRCS, consultant

Correspondence to: $\mathrm{Mr}$ N D Stafford.
}

questionnaires was supplemented by review of case notes and available radiographs.

Fisher's exact test was used to determine the significance of the association between handedness and the side of the neck on which symptoms and signs occurred, and also of the association between the side on which symptoms and signs occurred and radiographic or operative findings.

Sixty one questionnaires were returned, the response $(56 \%)$ probably reflecting the high mean age at initial presentation (63 years). Fifty five of the respondents were right handed and six left handed. Thirty three of the right handed patients and five of the left handed patients had experienced symptoms or signs, or both, on one side of the neck before treatment (the left side in 32 of the 33 right handed patients and the right side in all five left handed patients). The remaining patients had had only midline symptoms. In the patients with symptoms or signs on one side of the neck the association between that side and contralateral handedness was highly significant $(p<$ $0.0001)$.

Fully labelled radiographs were available for 26 patients. Of 12 with midline pouches without lateral extension radiographically, only one had had symptoms on one side of the neck and none had had signs on one side of the neck. Of 14 with pouches on one side of the neck radiographically (11 left, three right), all but one had had symptoms or signs, or both, on the side suggested by the radiographs. The exception had a left sided pouch but only midline dysphagia. The association between midline pouches or pouches on one side of the neck as predicted by signs and symptoms and as shown radiographically was highly significant $(p<0.0001)$.

\section{Discussion}

After emerging posteriorly in the midline through Killian's dehiscence pharyngeal pouches may remain in the midline or may pass laterally to one side of the oesophagus. Patients with such pouches have long been recognised as presenting much more commonly with left than with right sided symptoms. ${ }^{2}$ Although endoscopic and surgical findings confirm the predominance of left sided pouches, ${ }^{3}$ in individual cases where physical signs in the neck are absent it is often hard to establish whether the pouch is on one side even at operation. Additionally, the rarity of the condition means that large reported series such as this one are few and almost always represent the experience of several clinicians over a long period. This may explain why the question of left sided predominance has not previously been seriously 
addressed, although King suggested that anatomical asymmetry in the neck, particularly of the large vessels, may be a contributory factor. ${ }^{4}$

About $11 \%$ of the population is left handed, and $10 \%$ of our patients were left handed. Left handed people therefore seem as likely to develop pharyngeal pouches as right handed people. Symptoms are more likely than signs to occur predominantly on one side of the neck; the close correlation between our patients' signs and symptoms and the available radiograpns suggests that symptoms are an excellent indication of the site of the pouch anatomically. Our results also show a striking association between handedness and the occurrence of pharyngeal pouches on the opposite side. Limb and girdle musculature is greater on the dominant side, ${ }^{5}$ and we suggest that the enlarging pouch follows a path of least resistance to the contralateral side.

We conclude that symptoms that are predominantly on one side of the neck accurately predict lateral extension of a pharyngeal pouch, and that the side on which the pouch occurs is determined by the handedness of the patient.
We are indebted to the surgeons of the Royal National Throat, Nose and Ear Hospital for permission to study patients under their care, and to Mr A E Thompson and Mr H R Grant for allowing us to study their patients at St Thomas's and University College hospitals respectively.

\section{References}

1 Jones RFMcN. Hypopharyngeal diverticulum (pharyngeal pouch). In: Ballantyne J, Groves J, eds. Scott-Brown's diseases of the ear, nose and throat. 4th ed. Vol 4. London: Butterworths, 1979:210.

2 Butlin HT. On a second case of removal of a "pressure pouch" of the oesophagus. Br Med f 1898; : :8-10.

${ }^{3}$ Thompson StC. Diseases of the nose and throat. 1st ed. London: Waverley, 1911:566.

1 King BT. New concepts of the etiology and treatment of diverticula of the oesophagus. Surg Gynecol Obstet 1947;85:93-7.

${ }^{5}$ Chhibber SR. Determination of one sided upper limb dominance by measurement. Anatomischer Anzeiger 1972;130:S521-5.

(Accepted 12 January 1984)

\title{
Myocardial depression in streptococcal cellulitis
}

\author{
J D EDWARDS, P M SCHOFIELD
}

\begin{abstract}
A previously healthy woman developed streptococcal cellulitis of the leg after falling and lacerating her knee. On admission her blood pressure was unrecordable and her respiratory rate greater than 60 beats/minute. Mechanical ventilation was necessary, and plasma volume expansion was started, with little effect. Infusion of dopamine restored the blood pressure to $150 / 90 \mathrm{~mm} \mathrm{Hg}$, but interrupting the infusion for as little as 30 seconds resulted in profound hypotension. Insertion of a second central venous catheter allowed the dopamine to be given continuously, and the infusion was stopped successfully four days after her admission. She eventually made a complete recovery.

This case highlights the need for intensive supportive treatment in many streptococcal infections and, in particular, the need for inotropic support.
\end{abstract}

\section{Introduction}

Life threatening and fatal infections with Streptococcus pyogenes ( $\beta$ haemolytic streptococcus, Lancefield's group A) continue to occur despite the use of antibiotics. ${ }^{12}$ We describe a case of streptococcal cellulitis affecting the leg in a previously healthy patient.

\footnotetext{
University Hospital of South Manchester, West Didsbury, Manchester M20 8LR

J D EDWARDS, MB, MRCP, consultant physician, intensive care unit

P M SCHOFIELD, MB, MRCP, senior house officer

Correspondence to: Dr J D Edwards.
}

\section{Case report}

A 32 year old woman in her third pregnancy was admitted as an emergency at 32 weeks' gestation with colicky lower abdominal pain, thought to be due to labour. On admission she was moribund and unable to give a history because of severe dyspnoea. On examination she had central and peripheral cyanosis; the blood pressure was unrecordable, and the respiratory rate was greater than 60 beats $/ \mathrm{min}$. The left leg was swollen to the thigh. Because of suspected fetal distress an emergency caesarean section was performed. A dead fetus was delivered. She was transferred to the intensive care unit, and deep venous thrombosis and pulmonary embolism were provisionally diagnosed.

Ventilation with $70 \%$ oxygen was started, and blood gas analysis showed $\mathrm{pH} 7 \cdot 24$, oxygen tension $28.3 \mathrm{kPa}(212 \mathrm{~mm} \mathrm{Hg})$, and carbon dioxide tension $4 \mathrm{kPa}(30 \mathrm{~mm} \mathrm{Hg})$. Ventilation was continued and a Swan-Ganz catheter inserted. The mean pulmonary artery pressure was $15 \mathrm{~mm} \mathrm{Hg}$ and the pulmonary capillary wedge pressure $2 \mathrm{~mm} \mathrm{Hg}$. A chest $x$ ray film showed soft shadowing throughout both lung fields. Plasma volume expansion was started and the diagnosis reviewed. A small laceration was noted overlying the left patella. Cellulitis was suspected, and appropriate bacteriological specimens were sen:. Benzylpenicillin $4 \mathrm{MU}$ and flucloxacillin $1 \mathrm{~g}$ were administered intravenously.

After she had received 3.51 of intravenous fluid the pulmonary capillary wedge pressure was $18 \mathrm{~mm} \mathrm{Hg}$ and the blood pressure $60 \mathrm{~mm} \mathrm{Hg}$ systolic; she remained anuric. A dopamine infusion was started: $13 \mu \mathrm{g} / \mathrm{kg} / \mathrm{min}$ produced a blood pressure of $150 / 90 \mathrm{~mm} \mathrm{Hg}$ with a pulmonary capillary wedge pressure of $14 \mathrm{~mm} \mathrm{Hg}$ and a pulmonary artery pressure of $32 \mathrm{~mm} \mathrm{Hg}$, indicating that the circulatory failure was due to vasodilatation or myocardial depression or a combination of both.

She began to pass urine. Five hours after her admission to the intensive care unit her husband was available to provide a history. Thirty six hours previously she had fallen and lacerated her left knee while gardening, and subsequently the whole leg had become swollen and painful. Culture of fluid aspirated from beneath the lacerated area yielded a growth of haemolytic streptococcus (Lancefield's group A). Electrocardiography showed a normal tracing, apart from the rhythm, throughout her stay in hospital.

Mechanical ventilation had to be continued for 24 hours. An alarming feature was that whenever the infusion of dopamine was stopped for as little as 30 seconds-for instance, for injection of antibioticsshe developed circulatory collapse with a pulse rate of $15-20$ beats/min 